Ergod. Th. \& Dynam. Sys. (1981), 1, 381-388

Printed in Great Britain

\title{
Capacity of attractors
}

\author{
LAI-SANG YOUNG \\ Mathematics Institute, University of Warwick, England
}

(Received 13 March 1981 and revised 18 August 1981)

\begin{abstract}
Let $f$ be a diffeomorphism of a manifold and $\Lambda$ be an $f$-invariant set supporting an ergodic Borel probability measure $\mu$ with certain properties. A lower bound on the capacity of $\Lambda$ is given in terms of the $\mu$-Lyapunov exponents. This applies in particular to Axiom A attractors and their Bowen-Ruelle measure.
\end{abstract}

Let $f: M \circlearrowleft$ be a diffeomorphism of a manifold. Suppose $U \subset M$ is an open set with $f \bar{U} \subset U$. This tells us that there is an attracting set $\Lambda$ in $U$. A natural question is: how big is $\Lambda$ ? Intuitively the size of $\Lambda$ is related to the relative strengths of expansion and contraction of $f$ near this attracting set. We proceed to formulate a theorem in this direction.

First, we must decide on a notion of 'size'. Riemannian volume seems most natural, but attractors in 'dissipative' systems often have Riemannian measure zero. Hausdorff dimension is a classical tool for distinguishing between sets of measure zero. Our results are in terms of capacity, a notion very similar to Hausdorff dimension. Precise definitions are given later.

THEOREM. Let $f: M^{p} \bigcirc$ be a $C^{1+\alpha}(\alpha>0)$ diffeomorphism of a compact Riemannian manifold and let $\mu$ be an ergodic Borel probability measure on $M$ with Lyapunov exponents $\lambda_{1} \geq \cdots \geq \lambda_{p}$. Suppose also that $\lambda_{u}>0>\lambda_{u+1}$ and that $\mu$ has absolutely continuous conditional measures on unstable leaves. Then the lower capacity of the support of $\mu$,

$$
C(\operatorname{supp} \mu) \geq u+\frac{\lambda_{1}+\cdots+\lambda_{u}}{\left|\lambda_{p}\right|}
$$

COROllary. If $\Omega$ is an attractor of a $C^{2}$ Axiom $A$ diffeomorphism and $\mu$ is the Bowen-Ruelle measure $[1]$ on $\Omega$, then

$$
C(\Omega) \geq u+\frac{\lambda_{1}+\cdots+\lambda_{u}}{\left|\lambda_{p}\right|},
$$

where $\lambda_{1} \geq \cdots \geq \lambda_{p}$ are the $\mu$-exponents and $u=\operatorname{dim} E^{u}$.

This work is motivated by a conjecture of Yorke's [3], [4] and complements the work of Douady, Oesterlé [2] and Ledrappier [6] who have given upper bounds

† Address for correspondence: Lai-Sang Young, Department of Mathematics, Michigan State University, East Lansing, MI 48824, USA. 
for the Hausdorff dimension of attractors. Mallet-Paret [8], Manning [10], Mañé [9] and Takens [15] have also done work on the dimension of invariant sets.

The author thanks C. Robinson for the references in the proof of lemma 3 and the referee for his comments.

Remark. After this note was written the author found out that F. Ledrappier had independently arrived at a very similar result [7].

\section{Definitions}

Let $X$ be a compact metric space. For $\varepsilon>0$, let $N(\varepsilon)$ be the smallest number of $\varepsilon$-balls that cover $X$. Define the capacity or upper capacity of $X, \bar{C}(X)$ to be

$$
\limsup _{\varepsilon \rightarrow 0} \frac{\log N(\varepsilon)}{\log (1 / \varepsilon)}
$$

and the lower capacity of $X, C(X)$ to be

$$
\liminf _{\varepsilon \rightarrow 0} \frac{\log N(\varepsilon)}{\log (1 / \varepsilon)}
$$

(See for instance [3], [9] and [15].)

It is easy to verify that

$$
\begin{gathered}
d<\underline{C}(X) \Rightarrow \lim _{\varepsilon \rightarrow 0} N(\varepsilon) \varepsilon^{d}=\infty, \\
\underline{C}(X)<d<\bar{C}(X) \Rightarrow\left\{\begin{array}{l}
\limsup _{\varepsilon \rightarrow 0} N(\varepsilon) \varepsilon^{d}=\infty, \\
\liminf _{\varepsilon \rightarrow 0} N(\varepsilon) \varepsilon^{d}=0,
\end{array}\right.
\end{gathered}
$$

and

$$
d>\bar{C}(X) \Rightarrow \lim _{\varepsilon \rightarrow 0} N(\varepsilon) \varepsilon^{d}=0 .
$$

Recall that if $N(\varepsilon) \varepsilon^{d}$ is replaced by

$$
\alpha(\varepsilon, d)=\operatorname{Inf}_{\substack{\text { covers } \mathscr{A} \\ \operatorname{diam} \mathscr{A}<\varepsilon}} \sum_{A \in \mathfrak{Q}}(\operatorname{diam} A)^{d},
$$

then $\lim _{\varepsilon \rightarrow 0} \alpha(\varepsilon, d)$ always exists and the unique number $d_{0}$ with

$$
d<d_{0} \Rightarrow \lim _{\varepsilon \rightarrow 0} \alpha(\varepsilon, d)=\infty
$$

and

$$
d>d_{0} \Rightarrow \lim _{\varepsilon \rightarrow 0} \alpha(\varepsilon, d)=0
$$

is the Hausdorff dimension of $X$.

In general,

$$
H D(X) \leq C(X) \leq \bar{C}(X) .
$$

Examples of compact sets with strict inequalities can easily be constructed but $I$ do not know if these numbers coincide for nice invariant sets such as Axiom $\mathrm{A}$ basic sets. 
Capacity can also be defined in terms of separated sets. Let $S(\varepsilon)$ denote the maximum number of $\varepsilon$-separated points in $X$. Then $S(2 \varepsilon) \leq N(\varepsilon) \leq S(\varepsilon)$. Hence

and

$$
\bar{C}(X)=\limsup _{\varepsilon \rightarrow 0} \frac{\log S(\varepsilon)}{\log (1 / \varepsilon)},
$$

$$
C(X)=\liminf _{\varepsilon \rightarrow 0} \frac{\log S(\varepsilon)}{\log (1 / \varepsilon)} .
$$

Next we turn to the definition of Lyapunov exponents and stable manifolds. Let $f: M \circlearrowleft$ be a $C^{1+\alpha}$ diffeomorphism. Let $x \in M$ and $v \in T_{x} M$, the tangent space at $x$. Define the Lyapunov exponent of $f$ at $x$ in the direction $v$ to be the number

$$
\chi(x, v)=\lim _{n \rightarrow \infty}(1 / n) \log \left\|D f_{x}^{n} v\right\|
$$

if this limit exists.

Let $\mu$ be any $f$-invariant Borel probability measure. It is a fact that $\mu$-a.e. $x$ is 'regular', which implies in particular that $\chi(x, v)$ is well-defined for every $v \in T_{x} M$. For a regular point $x$, let

$$
\chi(x)=\max \{\chi(x, v): \chi(x, v)<0\} .
$$

Then

$$
W^{s}(x)=\left\{y \in M: \limsup _{n \rightarrow \infty}(1 / n) \log d\left(f^{n} x, f^{n} y\right) \leq \chi(x)\right\}
$$

is an immersed submanifold of $M$ called the stable manifold of $x$. The unstable manifold $W^{u}(x)$ is defined similarly with $f^{-1}$ playing the role of $f$. For a detailed account of this theory, see [12] or [14].

\section{Facts from Pesin theory}

In this section we fix some notations and recall some relevant facts. We follow mainly the approach in [11], a summary of which appears in [5].

Let $M$ be a $p$-dimensional compact Riemannian manifold and $f: M O$ be a $C^{1+\alpha}$ $(\alpha>0)$ diffeomorphism. Consider an ergodic Borel probability measure $\mu$ with Lyapunov exponents $\lambda_{1} \geq \cdots \geq \lambda_{p}$. Assume that $\lambda_{u}>0>\lambda_{u+1}$. Let

$$
\lambda=\min \left\{\lambda_{u},\left|\lambda_{u+1}\right|\right\}
$$

and fix small $\varepsilon>0$. For $l=1,2, \cdots$ let $\Lambda_{l}$ be the Pesin sets, that is,

$$
\begin{gathered}
\Lambda_{l}=\{x \in M: \forall m \in \mathbb{Z} \text { there is a splitting of the tangent space } \\
\qquad T_{f^{m_{x}}} M=E_{f^{m_{x}}}^{u} \oplus E_{f^{m_{x}}}^{s}
\end{gathered}
$$

satisfying the following conditions for all $n \geq 0, m \in \mathbb{Z}$ :

(1) $v \in E_{f^{m_{x}}}^{s} \Rightarrow\left\|D f_{f^{\prime \prime} x}^{n} v\right\| \leq l \exp \{\varepsilon|m|\} \exp \{-(\lambda-\varepsilon) n\}\|v\|$,

$$
\left\|D f_{f^{m} x}^{-n} v\right\| \geq(l \exp \{\varepsilon|m|\})^{-1} \exp \{(\lambda-\varepsilon) n\}\|v\|,
$$

(2) $v \in E_{f^{m} x}^{u} \Rightarrow\left\|D f_{f^{m} x}^{n} v\right\| \geq(l \exp \{\varepsilon|m|\})^{-1} \exp \{(\lambda-\varepsilon) n\}\|v\|$,

$$
\left\|D f_{f^{m} x}^{-n} v\right\| \leq l \exp \{\varepsilon|m|\} \exp \{-(\lambda-\varepsilon) n\}\|v\|
$$

and (3) $\left.\chi\left(E_{f^{m_{x}}}^{u}, E_{f^{m_{x}}}^{s}\right) \geq(l \exp \{\varepsilon|m|\})^{-1} \cdot\right\}$ 
It is well-known that

$$
\mu\left(\bigcup_{l} \Lambda_{l}\right)=1
$$

Some of our estimates are more transparent in 'Lyapunov charts'. 'Lyapunov charts' are non-autonomous changes in coordinates via which $f$ becomes uniformly hyperbolic. We collect a few facts that will be useful later.

Let $B_{r}^{u}=\left\{z \in \mathbb{R}^{u}:\|z\|<r\right\}$. For each fixed $l$ there are positive numbers $K_{l}$ and $\delta_{l}$ such that for every $x \in \Lambda_{l}$ there is a neighbourhood $B(x)$ of $x$ in $M$ and a diffeomorphism

$$
\psi_{x}: B_{1}^{u} \times B_{1}^{p-u} \rightarrow B(x)
$$

having the following properties:

(1) If $\langle\cdot, \cdot\rangle^{\prime}$ denotes the Riemannian metric on $M$ and $\langle\cdot, \cdot\rangle_{x}^{\prime}$ denotes the metric in $B(x)$ induced by $\psi_{x}$ from the Euclidean metric on $B_{1}^{u} \times B_{1}^{p-u}$, then

$$
K \leq \frac{\|\cdot\|_{x}^{\prime}}{\|\cdot\|^{\prime}} \leq K_{l}
$$

for some universal constant $K$.

(2) Let $W_{\text {loc }}^{u}(x)$ be the component of $W^{u}(x) \cap B(x)$ containing $x$. In the $x$-chart, $\psi_{x}^{-1} W_{\mathrm{loc}}^{u}(x)$ is the graph of some

$$
g_{x}: B_{1}^{u} \rightarrow B_{1}^{p-u}
$$

with $g(0)=0$ and $\|D g\| \leq \frac{1}{1000}$. The analogous statement for $W_{\mathrm{loc}}^{s}(x)$ also holds.

(3) If $x, y \in \Lambda_{l}$ and $d(x, y)<\delta_{l}$, then

$$
\psi_{x}^{-1} W_{\mathrm{loc}}^{u}(y) \cap B_{\frac{1}{2}}^{u} \times B_{1}^{p-u}
$$

is the graph of some $g: B_{\frac{1}{2}}^{u} \rightarrow B_{1}^{p-u}$ with $\|D g\| \leq \frac{1}{100}$.

\section{Lemmas}

Let $m$ denote Riemannian measure on $M$. If $L \subset M$ is an embedded disk then $L$ inherits from $M$ a Riemannian structure and hence a Riemannian measure. Let $m_{L}$ denote this Riemannian measure on $L$. For $x \in \Lambda_{I}$ and small $\rho>0$, let

$$
D_{\rho}^{u}(x)=\left\{y \in W_{\mathrm{loc}}^{u}(x): d(x, y)<\rho\right\}
$$

and

$$
D_{\rho}^{s}(x)=\left\{y \in W_{\mathrm{loc}}^{s}(x): d(x, y)<\rho\right\} .
$$

LEMMA 1. For each $l, \exists A_{l}>0$ s.t. for every $x \in \Lambda_{l}$ and $\rho<1 / K_{l}$, we have

$$
m_{W_{\text {loc }(x)}^{u}} D_{\rho}^{u}(x) \leq A_{l} \rho^{u} .
$$

Proof. This follows from the fact that $\psi_{x}^{-1} D_{\rho}^{u}(x)$ is contained in the graph of $g_{x} \mid B_{K_{i p}}^{u}$ $\operatorname{Vol}\left(B_{K_{l} \rho}^{u}\right) \sim \rho^{u}$ and that Riemannian metric is uniformly equivalent to $x$-chart metrics for all $x \in \Lambda_{l}$.

LEMMA 2. For each $l, \exists B_{l}>0$ s.t. for $\rho>0$ and $x, y \in \Lambda_{l}$ with $d(x, y)<\delta_{l}$, if

$$
W_{\text {loc }}^{u}(y) \cap D_{\rho}^{s}(x)=\varnothing
$$


then

$$
d(x, y) \geq B_{l} \rho .
$$

Proof. Again this is clear in the $x$-chart from the slopes of $W_{\text {loc }}^{u}(x)$ and $D_{\rho}^{s}(x)$ and the result follows from the equivalence of metric.

LEMMA 3. For every $\varepsilon>0$ and $\mu$-a.e. $x \in M, \exists C(x), \delta(x)>0$ s.t. for all $y \in D_{\delta(x)}^{s}$,

$$
d\left(f^{n} x, f^{n} y\right) \geq C(x) \exp \left\{\left(\lambda_{p}-\varepsilon\right) n\right\} d(x, y)
$$

for all $n \geq 0$. ( $\lambda_{p}=$ the smallest $\mu$-exponent. $)$

Proof. One way of seeing this is via theorem 4.1 (inequality (4.5) in particular) and the proof of theorem 5.1 of [14].

For small $\varepsilon>0$, let

$$
\begin{aligned}
\Gamma(l, C, \delta)= & \left\{x \in \Lambda_{l}: C(x) \geq C, \delta(x) \geq \delta\right. \text { and } \\
& \left.\left|\operatorname{Det}\left(D f^{n} \mid E_{x}^{u}\right)\right| \geq l^{-1} \exp \left\{\left(\lambda_{1}+\cdots+\lambda_{u}-\varepsilon\right) n\right\}, \forall n \geq 0\right\} .
\end{aligned}
$$

Choose $l, C$ and $\delta$ so that $\Gamma_{0}=\Gamma(l, C, \delta)$ has positive $\mu$-measure. Let $\tilde{\Gamma}_{0} \subset \Gamma_{0}$ be compact and have positive measure. Assume also that, for every $x \in \tilde{\Gamma}_{0}$,

$$
(1 / n) \sum_{i=0}^{n-1} \chi_{\Lambda_{l}} f^{i} x \rightarrow \mu \Lambda_{l} \text {. }
$$

LEMMA 4. For $n=1,2, \cdots, \exists \Gamma_{n} \subset \tilde{\Gamma}_{0}$ s.t.

(1) $\forall x \in \Gamma_{n}, \exists n \leq m_{n}(x)<n(1+\varepsilon)$ with

$$
f^{m_{n}(x)} x \in \Lambda_{l}
$$

(2) $\lim _{n \rightarrow \infty} \mu \Gamma_{n}=\mu \tilde{\Gamma}_{0}$.

Proof. This proof is borrowed from [5]. Let

$$
\begin{array}{r}
\Gamma_{n}=\left\{x \in \tilde{\Gamma}_{0}: \sum_{k=0}^{n-1} \chi_{\Lambda_{l}}\left(f^{k} x\right)<n \mu \Lambda_{l}\left(1+\frac{1}{3} \varepsilon\right)\right. \\
\text { and } \left.\sum_{k=0}^{n(1+\varepsilon)} \chi_{\Lambda_{l}}\left(f^{k} x\right)>n \mu \Lambda_{l}\left(1+\frac{2}{3} \varepsilon\right)\right\} .
\end{array}
$$

Next we consider a chart $\phi: B^{u} \times S \rightarrow M$, where $B^{u}$ is a closed $u$-dimensional disk, $S \subset B^{s}$ is compact, $\phi$ is continuous, $\phi \mid B^{u} \times\{y\}$ is $C^{1}$ for each $y \in S$, this derivative in the first variable is continuous on $B^{u} \times S$, and $\phi$ takes each fibre $B^{u} \times\{y\}$ into $W_{\text {loc }}^{u}(x)$ for some $x \in \tilde{\Gamma}_{0}$. We require also that $V=($ Image $\phi) \cap \tilde{\Gamma}_{0}$ has positive $\mu$-measure.

LEMMA 5. $\exists D>0$ with the property that, for large enough $n \in \mathbb{Z}^{+}, \exists x_{n} \in \tilde{\Gamma}_{0}$ s.t. if $L_{n}=W_{\text {loc }}^{u}\left(x_{n}\right)$ then

$$
m_{L_{n}}\left(\Gamma_{n} \cap L_{n}\right) \geq D \text {. }
$$

Proof. Let $\tilde{\mu}=\phi^{*}(\mu \mid V)$. Decompose $\tilde{\mu}$ into a transverse measure $\tilde{\mu}_{T}$ on $S$ and leaf measures $\tilde{\mu}_{y}$ on $B^{u} \times\{y\}$. Since $\mu$ has absolutely continuous conditional measures on $W^{u}$-leaves,

$$
\tilde{\mu} \ll \tilde{\mu}_{T} \times \lambda,
$$


where $\lambda$ is Lebesgue measure on $B^{u}$. It follows from this and $\mu\left(\Gamma_{n} \cap V\right) \rightarrow \mu V$ that

$$
\left(\tilde{\mu}_{T} \times \lambda\right)\left(\phi^{-1}\left(V \cap \Gamma_{n}\right)\right)
$$

is bounded away from zero. Hence the desired result.

LEMMA 6. $\exists N$ s.t. $\forall n \geq N, \exists n \leq m_{n}<n(1+\varepsilon), D>0, L_{n}=W_{\text {loc }}^{u}\left(x_{n}\right)$, some $x_{n} \in \tilde{\Gamma}_{0}$ and $B_{n} \subset L_{n} \cap \Gamma_{n}$ s.t.

(1) $\forall x \in B_{n}, f^{m_{n}} x \in \Lambda_{l}$;

(2) $m_{L_{n}} B_{n} \geq(1 / n \varepsilon) D$.

Proof. Choose $D$ and $L_{n}$ as in lemma 5. Partition $L_{n} \cap \Gamma_{n}$ by return time, i.e. for $n \leq j<n(1+\varepsilon)$ let

$$
R_{n j}=\left\{x \in L_{n} \cap \Gamma_{n}: f^{j} x \in \Lambda_{l}\right\}
$$

Then for some $j$,

$$
\begin{aligned}
m_{L_{n}} R_{n j} & \geq(1 / n \varepsilon) m_{L_{n}}\left(L_{n} \cap \Gamma_{n}\right) \\
& \geq(1 / n \varepsilon) D .
\end{aligned}
$$

Let this $j=m_{n}$ and $\boldsymbol{R}_{n j}=\boldsymbol{B}_{n}$.

\section{Proof of the theorem}

Recall that

$$
S(\alpha)=\text { maximum number of } \alpha \text {-separated points in supp }(\mu) .
$$

We fix some arbitrarily small $\varepsilon>0$; choose $\Gamma_{0}=\Gamma(l, C, \delta)$ and then $\tilde{\Gamma}_{0}$ and $\left\{\Gamma_{n}\right\}_{n=1,2, \cdots}$ as in lemma 4 . Let $D>0, L_{n}$ and $B_{n}$ be as in lemmas 5 and 6 . Choose $\delta_{0} \leq \delta$ small enough that $\forall x \in B_{n}$,

$$
D_{\delta_{0}}^{s}(x) \cap \bigcup_{y \in B_{n}} W_{\text {loc }}^{u}(y)=\{x\} .
$$

This is possible because $\tilde{\Gamma}_{0}$ is compact and uniformly hyperbolic and $W_{\mathrm{loc}}^{u}\left(\tilde{\Gamma}_{0}\right)$ and $W_{\text {loc }}^{s}\left(\tilde{\Gamma}_{0}\right)$ are continuous families of disks.

For large $n$, we construct a set $S_{n}$ as follows. Start with an arbitrary point $z_{1} \in f^{m_{n}} B_{n}$. Let $\rho=\exp \left\{\lambda_{p} m_{n}\right\}$ and pick

$$
z_{i} \in f^{m_{n}} B_{n}-\left(D_{\rho}^{u}\left(z_{1}\right) \cup \cdots \cup D_{\rho}^{u}\left(z_{i-1}\right)\right) \quad \text { for } i=2,3, \cdots
$$

until the process cannot be continued. Let $S_{n}$ consist of these $z_{i}$ 's. We know that

$$
\operatorname{card} S_{n} \geq \frac{\exp \left\{\left(\lambda_{1}+\cdots+\lambda_{u}-\varepsilon\right) m_{n}\right\} D}{l A_{l} \exp \left\{\lambda_{p} m_{n} u\right\} n \varepsilon}
$$

because

by lemma 1 , and

$$
m_{f^{m_{L_{n}}}} D_{\rho}^{u}\left(z_{i}\right) \leq A_{l} \rho^{u}
$$

$$
m_{f^{m_{n} L_{n}}} f^{m_{n}} B_{n} \geq l^{-1} \exp \left\{\left(\lambda_{1}+\cdots+\lambda_{u}-\varepsilon\right) m_{n}\right\}(D / n \varepsilon),
$$

since $B_{n} \subset \Gamma_{n} \subset \Gamma_{0}$.

Claim. For large $n$,

$$
x, y \in S_{n} \Rightarrow d(x, y) \geq \min \left\{\exp \left\{\lambda_{p} m_{n}\right\}, B_{l} C \delta_{0} \exp \left\{\left(\lambda_{p}-\varepsilon\right) m_{n}\right\}\right\} .
$$


Let $x, y \in S_{n}$. Since

$$
f^{m_{n}} D_{\delta_{0}}^{s}\left(f^{-m_{n}} x\right) \supset D_{C \exp \left\{\left(\lambda_{p}-\varepsilon\right) m_{n}\right\} \delta_{0}}^{s}(x)
$$

we have

$$
W_{\text {loc }}^{u}(y) \cap D_{C \exp \left\{\left(\lambda_{p}-\varepsilon\right) m_{n}\right\} \delta_{0}}^{s}(x)=\{x\} \text { or } \varnothing .
$$

Thus if $y$ is very near $x$, then either $y \in W_{\text {loc }}^{u}(x)$ in which case

$$
d(x, y) \geq \exp \left\{\lambda_{p} m_{n}\right\}
$$

by construction of $S_{n}$ or the intersection above is empty and lemma 2 applies to give

$$
d(x, y) \geq B_{l} C \delta_{0} \exp \left\{\left(\lambda_{p}-\varepsilon\right) m_{n}\right\} .
$$

To sum up, if

$$
E=\min \left\{1, B_{l} C \delta_{0}\right\}
$$

and

$$
\varepsilon_{n}=E \exp \left\{\left(\lambda_{p}-\varepsilon\right) n(1+\varepsilon)\right\}
$$

then

$$
S\left(\varepsilon_{n}\right) \geq \frac{\exp \left\{\left(\lambda_{1}+\cdots+\lambda_{u}-\varepsilon\right) m_{n}\right\} D}{l A_{l} \exp \left\{\lambda_{p} m_{n} u\right\} n \varepsilon},
$$

where $E, D, l$ and $A_{l}$ are independent of $n$. Thus

$$
\begin{aligned}
\liminf _{n \rightarrow \infty} \frac{\log S\left(\varepsilon_{n}\right)}{\log \left(1 / \varepsilon_{n}\right)} & \geq \lim _{n \rightarrow \infty} \frac{\left(\lambda_{1}+\cdots+\lambda_{u}-\varepsilon-\lambda_{p} u\right)+\left(1 / m_{n}\right) \log \left\{D /\left(l A_{l} \varepsilon n\right)\right\}}{-\left(1 / m_{n}\right) \log E-\left(\lambda_{p}-\varepsilon\right)} \\
& =\frac{\lambda_{1}+\cdots+\lambda_{u}-\varepsilon-\lambda_{p} u}{-\left(\lambda_{p}-\varepsilon\right)} .
\end{aligned}
$$

Now

$$
\frac{\log \varepsilon_{n}}{\log \varepsilon_{n+1}} \rightarrow 1 \quad \text { as } n \rightarrow \infty
$$

and for $\varepsilon_{n+1}<\tilde{\varepsilon}<\varepsilon_{n}$,

$$
\frac{\log S(\tilde{\varepsilon})}{\log (1 / \tilde{\varepsilon})} \geq \frac{\log S\left(\varepsilon_{n}\right)}{\log \left(1 / \varepsilon_{n}\right)} \cdot \frac{\log \left(1 / \varepsilon_{n}\right)}{\log \left(1 / \varepsilon_{n+1}\right)} .
$$

Letting $\varepsilon \rightarrow 0$ we complete the proof.

\section{Final remarks}

(1) The assumption that $\mu$ has absolutely continuous conditional measures on $W^{u}$-leaves implies that the $\mu$-exponents are (theoretically) observable, that is, there is a set $A \subset M$ with $m A>0$ such that for all $x$ in $A$, forward iterates of $f$ along the trajectory of $x$ give the $\mu$-exponents. This follows from the fact that

$$
m\left(\bigcup_{x \mu \text {-regular }} W^{s}(x)\right)>0
$$

which in turn follows from the absolute continuity of the $W^{s}$-foliation [12], [13].

(2) Even in the case of attractors, Lyapunov exponents do not reflect the dimension of the attractor if the underlying measure is too singular. An extreme example in the direction is the well-known figure 8 (see for instance [5]). 
(3) For a general lower bound such as we have here one cannot hope to include the other negative exponents. For example, consider $\Lambda=T^{2}$ in a 3-manifold with $f \mid \Lambda=a$ linear Anosov diffeomorphism with exponents $\lambda$ and $-\lambda$. Suppose that normal to $\Lambda$ we have a contraction with exponent $\gamma$ where $|\gamma|<\lambda$. Clearly

$$
C(\Lambda)=1+\frac{\lambda}{|-\lambda|},
$$

and $\gamma$ plays no role in the dimension of $\Lambda$. We do not know whether generically sharper estimates involving the other negative exponents can be given.

This research was partially supported by NSF Grant MCS-8002781.

\section{REFERENCES}

[1] R. Bowen. Equilibrium states and the ergodic theory of Anosov diffeomorphisms. Lecture Notes in Math. no. 470. Springer: Berlin, 1975.

[2] A. Douady \& J. Oesterlé. Dimension de Hausdorff des attracteurs. Comptes Rendus des Séances de L'Academie des Sciences 24 (1980), 1135-1138.

[3] P. Frederickson, J. Kaplan \& J. Yorke. The dimension of the strange attractor for a class of difference systems. Preprint.

[4] J. Kaplan \& J. Yorke. Chaotic behavior of multidimensional difference equations. Lecture Notes in Math. no. 730, pp. 228-237. Springer: Berlin, 1979.

[5] A. Katok. Lyapunov exponents, entropy and periodic orbits for diffeomorphisms. Publ. Math. I.H.E.S. 51 (1980), 137-174.

[6] F. Ledrappier. Some relations between dimension and Lyapunov exponents. Comm. Math. Phys. 81 (1981), 229-238.

[7] F. Ledrappier. In preparation.

[8] J. Mallet-Paret. Negatively invariant sets of compact maps and an extension of a theorem of Cartwright. Journal Diff. Eqtns. 22 (1976), 331-348

[9] R. Mañé. On the dimension of the compact invariant sets of certain nonlinear maps. Warwick Symp. Proc. Lecture Notes in Math. Springer: Berlin (to appear).

[10] A. Manning. Hausdorf dimension of horseshoes. In preparation.

[11] Ja. Pesin. Families of invariant manifolds corresponding to nonzero characteristic exponents. Math. of the USSR-Izvestija 10 (1976), 6, 1261-1305.

[12] Ja. Pesin. Characteristic Lyapunov exponents and smooth ergodic theory. Russ. Math. Surveys 32 (1977), 4, 55-114.

[13] C. Pugh \& M. Shub. Ergodic attractors. In preparation.

[14] D. Ruelle. Ergodic theory of differentiable dynamical systems. Publ. Math. I.H.E.S. 50 (1979), 27-58.

[15] F. Takens. Detecting strange attractors in turbulence. Warwick Symp. Proc. Lecture Notes in Math. Springer: Berlin (to appear). 